Draft version OCtober 8, 2018

Preprint typeset using $\mathrm{I}_{\mathrm{A}}^{\mathrm{A}} \mathrm{E} \mathrm{X}$ style emulateapj v. 5/2/11

\title{
STELLAR ROTATION EFFECTS IN POLARIMETRIC MICROLENSING
}

\author{
Sedighe SAJAdian ${ }^{1}$ \\ Draft version October 8, 2018
}

\begin{abstract}
It is well known that the polarization signal in microlensing events of hot stars is larger than that of main-sequence stars. Most hot stars rapidly rotate around their stellar axes. The stellar rotation makes ellipticity and gravity-darkening effects which break the spherical symmetry of the source shape and the circular symmetry of the source surface brightness respectively. Hence, it causes a net polarization signal for the source star. This polarization signal should be considered in polarimetry microlensing of fast rotating stars. For moderate rotating stars, lensing can magnify or even characterize small polarization signals due to the stellar rotation through polarimetry observations. The gravity-darkening effect due to a rotating source star makes asymmetric perturbations in polarimetry and photometry microlensing curves whose maximum happens when the lens trajectory crosses the projected position of the rotation pole on the sky plane. The stellar ellipticity makes a time shift (i) in the position of the second peak of the polarimetry curves in transit microlensing events and (ii) in the peak position of the polarimetry curves with respect to the photometry peak position in bypass microlensing events. By measuring this time shift via polarimetry observations of microlensing events, we can evaluate the ellipticity of the projected source surface on the sky plane. Given the characterizations of the FOcal Reducer and low dispersion Spectrograph (FORS2) polarimeter at Very Large Telescope (VLT) telescope, the probability of observing this time shift is so small. The more accurate polarimeters of the next generation may likely measure these time shifts and evaluate the ellipticity of microlensing source stars.
\end{abstract}

Subject headings: Gravitational lensing: micro, techniques: polarimetric, stars: steller rotation.

\section{INTRODUCTION}

Stellar rotation refers to the angular motion of a star around its axis. If there is no stellar rotation, the gravitational force condenses celestial bodes into perfect spheres. Whereas if an object rotates around its axis, some portion of the gravitational attraction provides the centrifugal acceleration whose value depends on the stellar latitude and decreases by increasing it from the stellar equator to the stellar pole. Therefore, the stellar rotation creates stellar oblateness (Collins \& Harrington 1966, Lebovitz 1967). For rapidly rotating stars the polar surface brightness is more than the equatorial one, the so-called gravity-darkening effect. This effect is resulted from the von Zeipel (1924)'s theorem, i.e. the radiative flux is proportional to the local effective gravity.

For main-sequence stars the stellar angular velocity $\Omega$ decreases with the stellar age t: $\Omega \propto \mathrm{t}^{-1 / 2}$, i.e. the Skumanich's relationship (Skumanich 1972, Durney \& Latour 1978). According to this relation, the star age can be derived by the rotational rate (Barnes 2007). Another result of this relation is that the rotational velocities of pre-main sequence stars are higher than those of main sequence stars. The stars in the spectral class between F5 up to O5 often rotate fast with the mean rotational velocity of order of $100-200 \mathrm{~km} \mathrm{~s}^{-1}$ (McAlister et al. 2005. Peterson et al. 2004). Also, their rotational velocity increases by mass and it maximizes for massive B-class

sajadian@ipm.ir

${ }^{1}$ Department of Physics, Sharif University of Technology, P.O. Box 11155-9161, Tehran, Iran stars. The less massive stars have much lower rotational speeds about a few $\mathrm{km} \mathrm{s}^{-1}$ after a few $100 \mathrm{Myr}$ (Kraft 1970, Gallet \& Bouvier 2013), since the magnetized stellar winds over the surface of these stars transport the angular momentum (Schatzman 1962). However, Irwin et al. (2011) by measuring the rotational period of stars with masses less than $0.35 \mathrm{M}_{\odot}$ found some exceptionally fast and slow rotators. Also, brown dwarfs have the averaged period of order of 15 hours at young ages (Scholz \& Eislöffel 2004, 2005).

The stellar rotation is determined via several methods. The projected radial velocity of rotating stars can be measured by spectroscopy observations of the Doppler broadening in the absorption lines of stars (Abney 1877). However, this method is not convenient for slowly rotating stars with the projected radial velocity less than $20 \mathrm{~km} \mathrm{~s}^{-1}$ (Bouvier 2013). For nearby fast rotating stars, the stellar oblateness projected on the sky plane can be measured by the interferometry method, e.g. Kervella et al. (2004). Combining the spectroscopy and interferometry methods helps us to charcterize the inclination angle of rotational axis with respect to the sky plane, e.g. Le Bouquin et al. (2009). The rotational periods of nearby spotted stars can also be determined via photometry observations of their light curves, e.g. Affer et al. (2012) and McQuillan et al. (2013). In that case, the stellar spots on rotating stars disturb the star light curves periodically. This method was first used to measure the Sun rotational period by Galileo Galilei (Casas et al. 2006). Generally, the mentioned methods can identify the rotational properties of nearby or bright (and massive) stars. 
Gravitational microlensing is a useful tool to highlight the rotational properties of distant stars. One channel is photometry observations of these events. In that case, the ellipticity or the radial velocity of source stars can perturb microlensing light curves, some aspects of this subject were studied by a number of authors. Heyrovský \& Loeb (1997) introduced an efficient method for calculating the microlensing light curve of an elliptical source by a point-mass lens and studied some properties of these light curves. The projected radial velocity of source stars or even the Einstein angular radius can be measured by spectroscopic observations of highmagnification microlensing events (Maoz \& Gould 1994 Gould 1997). Also Gaudi \& Haiman (2004) studied microlensing of elliptical sources by fold caustics and concluded that the ellipticity deviation in microlensing light curves is qualitatively similar to that due to the limb-darkening effect. In these references, the gravitydarkening effect resulted from the stellar ellipticity has not been considered.

In this work, we study the possibility to detect and characterize the rotational properties of distant source stars by performing polarimetry observations of highmagnification microlensing events. Rotating stars are oblate objects and their projected surface brightness will not be symmetric owing to the projection process unless their rotation axis orients toward the observer. Consequently, a rotating star has a net polarization signal whose value is a function of the stellar angular velocity and the inclination angle of its rotation axis. Lensing can magnify these small polarization signals and cause them to be realized or even characterized through polarimetry observations.

In section (2), we explain the formalism used for calculating the polarization signal of an elliptical source star. The properties of polarimetry microlensing events of elliptical sources will be studied in the next section. In section (4), we first study the statistic of fast rotating stars using the Kepler data. Then, we do a Monte Carlo simulation of high-magnification microlensing events of rotating stars toward the Galactic spiral arms to evaluate the efficiency for detecting the rotation-induced (polarimetry and photometry) perturbations of source stars. Finally, we conclude in the last section.

\section{THE POLARIZATION SIGNAL OF AN ELLIPTICAL SOURCE}

The intrinsic polarization signal of rapidly rotating early-type stars was first studied by Harrington \& Collins (1968). Also, the scattering polarization generated by nonradial pulsating stars was calculated by Stamford \& Watson (1980). Bjorkman \& Bjorkman (1994) have analytically calculated the polarization signal due to a fast rotating star surrounded by an axisymmetric disk. AlMalki et al. (1999) and Ignace et al. (2009) estimated the Stokes parameters due to anisotropic light sources with spherical envelopes and envelopes of arbitrary shapes by ignoring the finite size effect of the source star. Recently, several codes were written to solve the coupled problem of the Non-Local Thermodynamic Equilibrium (NLTE) and radiative equilibrium for arbitrary three-dimensional envelope geometries, using the Monte Carlo method ( e.g. Carciofi \& Bjorkman 2006, Carciofi et al. 2006, Whitney et al. 2013).
We use the formalism developed by Harrington \& Collins (1968) to calculate the intrinsic polarization signals of rotating stars. Accordingly, we assume that (i) The stellar envelope is optically thin enough to use the single scattering approximation. (ii) The rotational angular velocity is constant over the stellar surface and there is no differential rotation effect (see e.g. Kitchatinov 2005). (iii) The stellar angular momentum is not transported from the stellar surface during microlensing events. (iv) The star is rotating as a rigid body so that the von Zeipel's theorem is applicable. (v) The magnetic field of the source star is negligible. (vi) There is no disk around the source star. (vii) The angular rotation velocity normalized to the break-up velocity of the source star is small enough to approximate the local polarization over the rotating source surface with the local polarization over a spherical source surface. Note that fast rotating stars, e.g. B-class stars, are intrinsically variable and are not suitable candidates to be studied for microlensing observations.

We consider some parameters to describe an elliptical source: (i) the stellar equatorial and polar radii $\mathrm{R}_{\text {eq }}$ and $R_{p}$. (ii) The inclination angle of the stellar rotation axis with respect to the sky plane i. (iii) Two parameters to represent the limb-darkening coefficients $c_{1}$ and $c_{2}$.

To describe an elliptical source in the sky plane we need two coordinate systems: (i) Observer coordinate frame $\left(\mathrm{x}_{\mathrm{O}}, \mathrm{y}_{\mathrm{o}}, \mathrm{z}_{\mathrm{O}}\right)$ so that the projected source center is at its origin, the observer is on the $z_{0}$-axis at $+\infty$ and the $z_{0}-y_{o}$ plane contains the stellar rotation axis (i.e. its $\mathrm{x}_{\mathrm{O}}$-axis is parallel with the semimajor axis of the projected elliptical source). (ii) Stellar coordinate system $\left(\mathrm{x}_{\star}, \mathrm{y}_{\star}, \mathrm{z}_{\star}\right)$ so that the $\mathrm{y}_{\star}$-axis is along the stellar rotation axis and the $x_{\star}$-axis is along the observer's $x_{0}$-axis. We transform the second coordinate system to the first one by a rotation around $\mathrm{x}_{\star}$-axis by the inclination angle $-\mathrm{i}^{\circ}$, so that:

$$
\begin{aligned}
& x_{o}=x_{\star}, \\
& y_{o}=y_{\star} \cos (i)-z_{\star} \sin (i), \\
& z_{o}=y_{\star} \sin (i)+z_{\star} \cos (i) .
\end{aligned}
$$

We use $\left(\mathrm{R}_{\star}, \theta_{\star}, \phi_{\star}\right)$ to represent points over the stellar surface in the spherical stellar coordinate, i.e.

$$
\begin{aligned}
x_{\star} & =R_{e q} \sin \left(\theta_{\star}\right) \sin \left(\phi_{\star}\right), \\
y_{\star} & =R_{p} \cos \left(\theta_{\star}\right), \\
z_{\star} & =R_{e q} \sin \left(\theta_{\star}\right) \cos \left(\phi_{\star}\right) .
\end{aligned}
$$

Noting that $\mathrm{R}_{\mathrm{eq}}$ can be determined according to the stellar angular velocity $\Omega$. Generally, $\mathrm{R}_{\star}=\sqrt{\mathrm{x}_{\star}^{2}+\mathrm{y}_{\star}^{2}+\mathrm{z}_{\star}^{2}}$ depends on $\theta_{\star}$ owing to the stellar oblateness (Collins \& Harrington 1966):

$$
R_{\star}\left(\omega, \theta_{\star}\right)=\frac{3 R_{p}}{w \sin \theta_{\star}} \cos \left[\frac{\pi+\cos ^{-1}\left(\omega \sin \theta_{\star}\right)}{3}\right],
$$

where $\omega=\Omega / \Omega_{\text {crit }}$ is the ratio of the star's angular velocity to the critical or break-up velocity $\Omega_{\text {crit }}$ at which the centrifugal force at the stellar equator becomes equal to the gravitational attraction, given by: $\Omega_{\text {cirt }}^{2}=(2 / 3)^{3} \mathrm{G} \mathrm{M}_{\star} / \mathrm{R}_{\mathrm{p}}^{3}$ where $\mathrm{M}_{\star}$ is the stellar mass and $\Omega_{\text {crit }}$ is measured in the unit of radian per second.

In our formalism, the projected position of the stel- 
lar rotation pole in the observer coordinate system is $\boldsymbol{d}_{\mathrm{R}}^{\dagger}=\left(0, \mathrm{R}_{\mathrm{p}} \cos (\mathrm{i}), 0\right)$. The emergent radiative flux of the source star in this point maximizes. The projection of the source star on the sky plane is an ellipse whose semimajor and minor axes are $a=R_{\text {eq }}$ and $b=\sqrt{R_{\text {eq }}^{2} \sin ^{2}(i)+R_{p}^{2} \cos ^{2}(i)}$ respectively. Also the $\mathrm{x}_{\mathrm{O}}$ and $\mathrm{y}_{\mathrm{o}}$-axes are toward its semimajor and minor axes. We use $(\rho, \phi)$ to represent points over the stellar surface projected on the sky plane in the polar observer coordinate where $\phi \in[-\pi, \pi]$ and $\rho \in\left[0, \rho_{\mathrm{m}}\right]$. $\rho_{\mathrm{m}}=\mathrm{b} / \sqrt{(\mathrm{b} \cos \phi)^{2}+(\mathrm{a} \sin \phi)^{2}}$ is normalized to $\mathrm{a}$ and in the range of $\rho_{\mathrm{m}} \in[\mathrm{b} / \mathrm{a}, 1]$.

To calculate the polarization signal of an elliptical star, we use the Stokes intensities. There are four Stokes intensities $\mathrm{I}_{\mathrm{I}}, \mathrm{I}_{\mathrm{Q}}, \mathrm{I}_{\mathrm{U}}$ and $\mathrm{I}_{\mathrm{V}}$ which represent the total intensity, two components of linear polarized intensities and circular polarized intensity over the source surface, respectively (Tinbergen 1996). Taking into account that there is only linear polarization of light scattered on the stellar atmosphere, we set $I_{V}=0$. The other intensities are estimated according to the limb-darkening effect producing a local polarization owing to the light scattering in the stellar atmosphere. This local polarization depends on the scatterer species and the nature of the source star (Ingrosso et al. 2012,2015). For example, in late-type main-sequence stars, the polarization signal is generated rather owing to Rayleigh scattering on neutral hydrogens and a bit due to Thompson scattering by free electrons (Fluri \& Stenflo 1999). In cool giant stars Rayleigh scattering on atomic and molecular species or on dust grains generates the polarization signal. In that case, Simmons et al. (2002) introduced the appropriate Stokes parameters for giant stars with spherical circumstellar envelopes lensed by a single lens. In hot early-type stars with a free electron atmosphere mostly Thompson scattering produces the polarization signal (Chandrasekhar 1960). The magnitude of Stokes (total and polarized) intensities over the surface of these stars can be written in the form (Schneider \& Wagoner 1987):

$$
\begin{aligned}
I_{I}(\rho, \phi) & =I_{0, o}(\rho, \phi)\left[1-c_{1}(1-\mu)\right], \\
I_{P}(\rho, \phi) & =I_{0, o}(\rho, \phi)\left[c_{2}(1-\mu)\right],
\end{aligned}
$$

where $\quad \mathrm{I}_{\mathrm{P}}=\sqrt{\mathrm{I}_{\mathrm{Q}}^{2}+\mathrm{I}_{\mathrm{U}}^{2}}, \quad \mathrm{c}_{1}=0.64, \quad \mathrm{c}_{2}=0.032$, $\mu=\sqrt{1-\rho^{2} / \rho_{\mathrm{m}}^{2}}$ and $\mathrm{I}_{0, \mathrm{o}}(\rho, \phi)$ is the emergent radiative flux of the source star in the observer coordinate system. Note that Chandrasekhar (1960) considered a spherically symmetric, isotropically scattering atmosphere to calculate the Stokes intensities. Indeed, we assume that the effect of the stellar rotation is small enough to adopt for rotating stars the local Stokes intensities in equation (4). We calculate the radiative flux $\mathrm{I}_{0, \mathrm{o}}$ in the observer coordinate system starting from the flux in the stellar coordinate system $\mathrm{I}_{0, \mathrm{~s}}$ and using the coordinate transformations in equation (1). The gravity-darkening effect which is a result of the stellar ellipticity causes the intrinsic radiative flux of the source star $I_{0, \mathrm{~s}}$ changes over the source surface. According to the von Zeipel (1924)'s theorem, we can express the stellar radiative flux as a function of the effective gravity $\mathrm{g}_{\text {eff }}$ over the surface of a uniformly rotating star (in the stellar coordinate) which is given by:

$$
I_{0, s}\left(\Omega, \theta_{\star}\right)=-\frac{L_{\star}(\mathcal{P})}{4 \pi G M_{\star}(\mathcal{P})}\left|\boldsymbol{g}_{e f f}\left(\Omega, \theta_{\star}\right)\right|,
$$

where the stellar luminosity $L_{\star}$ and the stellar mass $M_{\star}$ are evaluated on a surface of a constant pressure $\mathcal{P}$. If there is no angular momentum transport on the star surface, the vector of the effective gravity in the Roche model and in the hydrostatic equilibrium is given by (see e.g. Maeder \& Meynet 2011):

$$
\begin{aligned}
\boldsymbol{g}_{e f f}\left(\Omega, \theta_{\star}\right) & =\left[-\frac{G M_{\star}}{R_{\star}^{2}\left(\theta_{\star}\right)}+\Omega^{2} R_{\star}\left(\theta_{\star}\right) \sin ^{2} \theta_{\star}\right] \boldsymbol{e}_{r} \\
& +\left[\Omega^{2} R_{\star}\left(\theta_{\star}\right) \sin \theta_{\star} \cos \theta_{\star}\right] \boldsymbol{e}_{\theta},
\end{aligned}
$$

where $\boldsymbol{e}_{\mathrm{r}}$ and $\boldsymbol{e}_{\theta}$ are the unit vectors in the radial and latitudinal directions. Accordingly, $\mathrm{I}_{0, \mathrm{~s}}$ for an elliptical source is proportional to $\left|\boldsymbol{g}_{\text {eff }}\left(\Omega, \theta_{\star}\right)\right|$ and a function of $\left(\Omega, \theta_{\star}\right)$.

Also we assume that the stellar atmosphere has an elliptical shape due to the stellar rotation, so that polarization vectors are tangential to co-center ellipses whose centers coincide to the stellar center. The normal vector to the elliptical source surface in each point $\left(\mathrm{x}_{\mathrm{o}}, \mathrm{y}_{\mathrm{o}}\right)$ from the source center is given by: $\boldsymbol{n}=\left(\mathrm{x}_{\mathrm{o}} / \mathrm{a}^{2}, \mathrm{y}_{\mathrm{o}} / \mathrm{b}^{2}\right)$.

Now by integrating these Stokes intensities over the source surface, the corresponding Stokes parameters $\mathrm{S}_{\mathrm{I}}$, $\mathrm{S}_{\mathrm{Q}}$ and $\mathrm{S}_{\mathrm{U}}$ are obtained. If the source light is magnified by a microlens, we should add a weight function for each source surface element, i.e. the magnification factor A. In that case, the Stokes parameters are given by:

$$
\begin{aligned}
S_{I} & =\rho_{\star}^{2} \int_{-\pi}^{\pi} d \phi \int_{0}^{\rho_{m}} \rho d \rho I_{I}(\rho, \phi) A(u), \\
\left(\begin{array}{c}
S_{Q} \\
S_{U}
\end{array}\right) & =\rho_{\star}^{2} \int_{-\pi}^{\pi} d \phi \int_{0}^{\rho_{m}} \rho d \rho I_{P}(\rho, \phi) A(u)\left(\begin{array}{l}
-\cos 2 \varphi \\
-\sin 2 \varphi
\end{array}\right),
\end{aligned}
$$

where $\rho_{\star}=R_{\text {eq }} x_{\text {rel }} / R_{E}$ is the stellar equatorial radius projected on the lens plane and normalized to the Einstein radius $R_{E}, x_{r e l}=D_{l} / D_{s}$ is the ratio of the lens distance to the source distance from the observer position, $\mathrm{u}=\left|\boldsymbol{u}_{\mathrm{cm}}-\boldsymbol{\rho} \rho_{\star}\right|$ is the distance of each projected element over the source surface with respect to the lens position, $\boldsymbol{u}_{\mathrm{cm}}$ is the vector of the lens position from the source center and $\varphi$ is the angle of the normal vector to the co-center ellipse passing the point $(\rho, \phi)$ (i.e. $\boldsymbol{n}$ ) with respect to the $\mathrm{x}_{\mathrm{O}}$-axis. In equation (7), we have aligned the signs of two components of polarized Stokes intensities so that polarization vectors become tangential to the co-center ellipses. Finally, the polarization degree $\mathrm{P}$ and the angle of polarization $\theta_{\mathrm{p}}$ as functions of total Stokes parameters are (Chandrasekhar 1960):

$$
\begin{gathered}
P=\frac{\sqrt{S_{Q}^{2}+S_{U}^{2}}}{S_{I}}, \\
\theta_{p}=\frac{1}{2} \tan ^{-1} \frac{S_{U}}{S_{Q}} .
\end{gathered}
$$

If there is no lensing effect, the elliptical source has a net polarization signal whose magnitude depends on the inclination angle and its rotational speed. In Figure (1) we plot the net polarization signal of an elliptical 


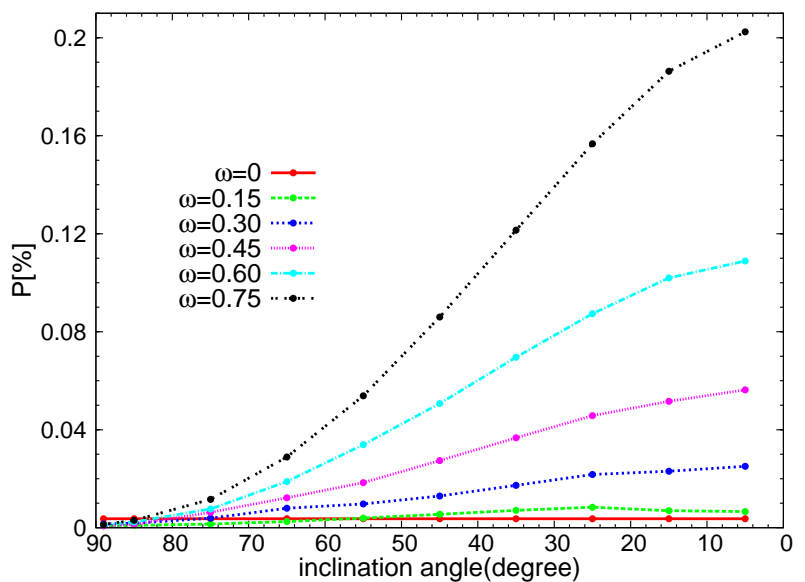

Figure 1. The net polarization signal of an elliptical source star versus the inclination angle of the rotation axis with respect the sky plane, for different values of $\omega$ i.e. the ratio of the stellar angular velocity to the critical velocity. The used parameters are $M_{\star}=5 M_{\odot}$ and $\mathrm{R}_{\mathrm{p}}=3.6 \mathrm{R}_{\odot}$.

source versus the inclination angle for different values of $\omega$. This plot is the same as Figure (4) of Harrington \& Collins (1968) representing the polarization signal of rotating stars. The inclination angle of the rotational axis in our formalism is the complementary angle to the one introduced by Harrington \& Collins. If $\mathrm{i}=90^{\circ}$, the projected shape of the source star on the sky plane is a perfect circle with the radius equals to $R_{\text {eq }}$. If $i=0^{\circ}$, the projected shape will be an ellipse whose semimajor and minor axes are $R_{e q}$ and $R_{p}$. Therefore, by decreasing the inclination angle the ellipticity of the source surface projected on the sky plane increases. On the other hand, faster rotating stars are more oblate. When $\omega=0$ the source star is a perfect sphere without any gravitydarkening effect. In that case, the inclination angle does not alter the net polarization signal. Noting that the stellar polar radius is determined using the mass-radius relation while its equatorial radius depends on $\omega$ (see equation 3). Also, the polarization angle of an elliptical source is zero with respect to its semimajor axis and it does not depend on the inclination angle and the stellar angular velocity.

According to Figure (1), the intrinsic polarization signal produced by an elliptical source is generally less than $0.2 \%$ and in the case of very fast rotating stars $(\omega>0.6)$ comparable with the polarization signal in the transit microlensing events for which $\mathrm{P} \simeq 0.6-0.7 \%$. Polarization signals of this amplitude can be measured directly. Therefore, the stellar rotating effects should be considered in the polarimetric microlensing calculations of fast rotating stars allowing to characterize the elliptical properties of the source stars. On the other hand, the intrinsic polarization signals for slow or moderate rotating stars with $\omega<0.6$ that are too small to be measured by themselves, may be magnified during a microlensing event. In the next section, we add the lensing effect and study the polarimetry microlensing of elliptical sources. Our aim is to investigate if these signals can be detected in polarimetry and photometry observations of high-magnification

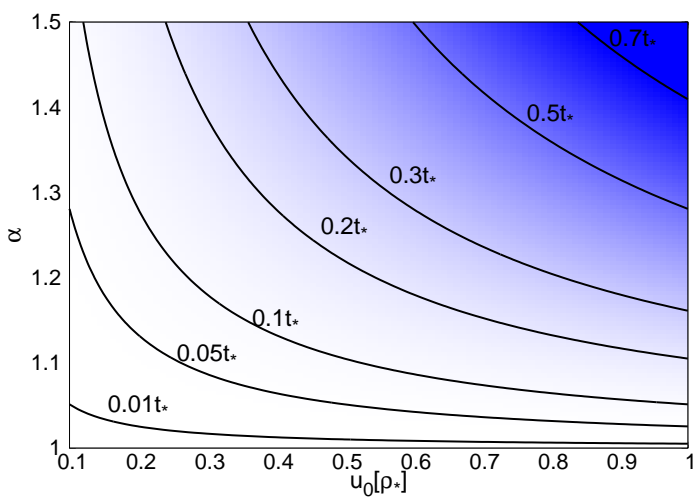

Figure 2. The contour lines of the time shift in polarimetry peak positions of transit microlensing events of elliptical source stars given by the equation (10), in the plane containing $\alpha$ (the ratio of the semimajor to semiminor axes of the projected source surface) and the lens impact parameter normalized to $\rho_{\star}$. The contour lines are in the unit of the time scale of crossing the source radius $t_{\star}$.

microlensing events.

\section{CHARACTERISTICS OF POLARIMETRIC MICROLENSING OF ELLIPTICAL SOURCES}

The stellar rotation causes (i) the ellipticity and (ii) the gravity-darkening effects both of which make perturbations in the light and polarimetric curves of microlensing events. Here, we aim to study these perturbations which are classified in the following subsections.

\subsection{Time shift in the polarimetry peak location}

In the case of spherically symmetric (non rotating) source stars, photometric and polarimetric light-curves in single-lens microlensing events have symmetric shapes with respect to the time $t_{0}$ of the closest approach. In the case of rotating source stars, the ellipticity effect breaks the symmetry of these curves around $t_{0}$.

In particular, (i) the location of the peak(s) in the transit and bypass polarimetry microlensing curves changes from the points $\mathrm{u} \approx 0.96 \rho_{\star}$ and $\mathrm{u}=\mathrm{u}_{0}$ respectively, and (ii) the location of the peak in light curves shifts from the time of the closest approach. But, we can not realize the shift in the position of the photometric peak from its true value (i.e. the time of the closest approach) by doing only photometry observation of microlensing events. While by performing polarimetry observations of transit microlensing events, we can discern the time shift in the position of the second peak with respect to the symmetric (with respect $t_{0}$ ) position of the first peak, since the symmetry in positions of polarimetry peaks breaks due to the stellar ellipticity. Also in transit microlensing events the ellipticity can shift the relative minimum location between two polarimetry peaks with respect to the photometry peak position. In bypass microlensing events, the time shift between the position of the polarimetry peak and the position of the photometry peak helps to distinguish the ellipticity effect. However, in the bypass polarimetry microlensing events with $\mathrm{u}_{0}$ so larger than $\rho_{\star}$ the rotation-induced perturbations becomes too small, because of the averaging process. 
As we shall see in subsection (3.2), gravity-darkening breaks the symmetry of the source surface brightness and affects on the peak position of polarimetry and light curves, unless $\mathrm{i}=90^{\circ}$. However, if the inclination angle is large enough so that the projected position of stellar pole on the sky plane (i.e. $\boldsymbol{d}_{\mathrm{R}}$ ) does not posit at the stellar limb, we can ignore the gravity-darkening effect for calculating the time shift in peak positions of polarimetry and light curves. In that case, by considering only the ellipticity effect we assess the time shift in the position of the second polarimetric peak. In transit microlensing events, the lens crosses the source edges at two moments which are given by:

$t_{1,2}\left[t_{E}\right]=\frac{-u_{0} \sin (2 \xi)\left(\alpha^{2}-1\right) \pm 2 \alpha \sqrt{b^{2} \cos ^{2} \xi+a^{2} \sin ^{2} \xi-u_{0}^{2}}}{2\left(\cos ^{2} \xi+\alpha^{2} \sin ^{2} \xi\right)}$

where $t_{1,2}\left[t_{E}\right]$ are in the unit of the Einstein crossing time $\mathrm{t}_{\mathrm{E}}, \xi$ is the angle between the lens trajectory and the source semimajor axis and $\alpha=\mathrm{a} / \mathrm{b}$ the ratio of the source semimajor to minor axes. Hence, the second peak in polarimetry curves with respect to the symmetric position of the first peak shifts due to the ellipticity by:

$$
\delta_{t}=\frac{u_{0}\left[\rho_{\star}\right] \sin (2 \xi)\left(\alpha^{2}-1\right)}{\left(\cos ^{2} \xi+\alpha^{2} \sin ^{2} \xi\right)} t_{\star},
$$

where $\mathrm{u}_{0}\left[\rho_{\star}\right]$ is the lens impact parameter in units of $\rho_{\star}$ and $\mathrm{t}_{\star}=\rho_{\star} \mathrm{t}_{\mathrm{E}}$ is the time scale for crossing the source radius. The time shift $\delta_{\mathrm{t}}$ maximizes when $\cos \xi=\alpha / \sqrt{\alpha^{2}+1}$. In Figure 22, we plot the contour lines of $\delta_{\mathrm{t}}$ in the plane containing $\mathrm{u}_{0}$ and $\alpha$, for values of the lens trajectory angles $\xi$ which offer the maximum values of $\delta_{t}$. This time shift is less than $t_{\star}$ by two or one order of magnitude.

To estimate the magnitude of $t_{\star}$ and $\delta_{t}$, we consider early-type stars as microlensing sources which most rotate fast. These stars have the effective temperature in the range of $\mathrm{T}_{\text {eff }} \in[7500: 30000] \mathrm{K}$ and the stellar polar radius in the range of $\mathrm{R}_{\mathrm{p}} \in[1.4: 6.6] \mathrm{R}_{\odot}$. It is well known that the abundance of early-type stars in the Galactic spiral arms is more than that in the Galactic bulge. Therefore, we consider hypothetical microlensing events toward the Galactic spiral arm and in the direction of $\mathrm{l}=300^{\circ}$ and $\mathrm{b}=-1^{\circ}$, i.e. the CarinaSagittarius arm. This arm mostly contains young stellar objects (e.g. Churchwell et al. 2009). By using a Monte Carlo simulation we estimate the mean lens mass $\mathrm{M}_{1}=0.3 \mathrm{M}_{\odot}$ from the Kroupa mass function (Kroupa 2001, Kroupa et al. 1993) and the average distances $\mathrm{D}_{\mathrm{l}}=4.0 \mathrm{kpc}$ and $\mathrm{D}_{\mathrm{s}}=8.5 \mathrm{kpc}$ of lenses and sources from the observer by using the angular distribution of stars in the Galactic bulge and disk. In this way the Einstein radius results to be $R_{E}=2.3 \mathrm{AU}$. Moreover, by adopting the synthetic Besançon model (Robin et al. 2003) we estimate the source star radius $\rho_{\star}$, which is in the range [0.001:0.006] (in units of $\mathrm{R}_{\mathrm{E}}$ ). Towards this Galactic spiral arm the averaged amount of the Einstein crossing time is longer than that toward the Galactic bulge and is about $\overline{\mathrm{t}}_{\mathrm{E}} \simeq 97$ days (Rahal et al. 2009). Consequently, for microlensing events toward this Galactic spiral arm the value of $t_{\star}$ will be in the range of $t_{\star} \in[2.3: 14.0]$ hours. Consider- ing a common value for the time shift $\delta_{\mathrm{t}} \sim 0.1 \mathrm{t}_{\mathrm{E}}$, it will be in the range $\delta_{\mathrm{t}} \in[14.0: 83.8] \mathrm{min}$.

To evaluate how many polarimetry data points can potentially be taken during this time shift $\delta_{t}$, we assume that these observations are done by the FOcal Reducer and low dispersion Spectrograph (FORS2) polarimeter at Very Large Telescope (VLT) telescope. The necessary exposure time for FORS2 to achieve the polarimetric accuracy $0.1 \%$ for a magnified star with the apparent magnitude about $\mathrm{m}_{\mathrm{I}}=14.5 \mathrm{mag}$ is about $8 \mathrm{sec}{ }^{2}$. In addition to the exposure time, there are two extra waste times due to the retarder waveplate rotation and CCD readout. Indeed, to accurately determine the polarization signal, the source flux should be measured in 16 $0^{2}$ directions from $0^{\circ}$ to $337.5^{\circ}$, in $22.5^{\circ}$ steps. The signal Q taken exposure time in all retarder waveplate positions should reach to 1000 to give up the polarimetry accuracy $0.1 \%$ (Ejeta et al. 2012). Rotating the retarder waveplate of FORS2 takes some time about $\sim 1 \mathrm{~min}$. On the other hand, the FORS2 CCD will be saturated after $2 \mathrm{sec}$ exposure time from a bright star with $\mathrm{m}_{\mathrm{I}}=14.5 \mathrm{mag}$. Therefore, the CCD detector should be read 4 times for taking every polarimetry data point with the polarimetry accuracy $0.1 \%$. The FORS2 CCD readout takes time 30 sec. Accordingly, we should add to the exposure time about $18 \mathrm{~min}$ as overhead time due to the retarder waveplate rotation and CCD readout. This overhead time does not depend on the magnification factor and the source brightness and is constant for taking each data point by FORS2 with the highest polarimetry accuracy. Thus, the total observational time for each polarimetry data point of a magnified source star with $\mathrm{m}_{\mathrm{I}}=14.5 \mathrm{mag}$ is about $\mathrm{T}_{\mathrm{obs}}=18.13 \mathrm{~min}$.

Consequently, If FORS2 uninterruptedly observers a transit microlensing event of an early-type star, it will averagely take $1-5$ data points during this time shift, where we estimate the number of possible data points by the factor $\delta_{\mathrm{t}} / \mathrm{T}_{\mathrm{obs}}$. This number is not sufficient to correctly realize $\delta_{\mathrm{t}}$. However, the factor $\delta_{\mathrm{t}} / \mathrm{T}_{\mathrm{obs}}$ increases in some specific microlensing events, e.g. when (i) the Einstein crossing time is very long, (ii) the lens crosses the source surface with large impact parameters (see Figure 2 and (iii) the projected radius of the source star normalized to the Einstein radius is large which happens e.g. when the lens and source stars are so close to each other i.e. $\mathrm{x}_{\mathrm{rel}} \sim 1$. Otherwise, the possibility of discerning this time shift is almost out of the present technology (FORS2 polarimeter at VLT telescope). However, this time shift can likely be realized by high-quality instruments of the next generation.

If the time shift $\delta_{\mathrm{t}}$ is measured from the polarimetric observations of microlensing events, we can estimate the parameter $\alpha=\mathrm{R}_{\text {eq }} / \sqrt{\mathrm{R}_{\text {eq }}^{2} \sin ^{2}(\mathrm{i})+\mathrm{R}_{\mathrm{p}}^{2} \cos ^{2}(\mathrm{i})}$ which is a degenerate function of the inclination angle and the intrinsic ellipticity of the source star, assuming the lens impact parameter and $\xi$ are carefully measured from photometry observations. The parameter $\alpha$ shows the ellipticity of the source surface projected on the sky plane. Noting that the uncertainties in the parameters $\mathrm{u}_{0}, \xi$ and $\delta_{\mathrm{t}}$ cause an uncertainty in the parameter $\alpha$. The uncer-

\footnotetext{
${ }^{2}$ http://www.eso.org/observing/etc/bin/gen
} 


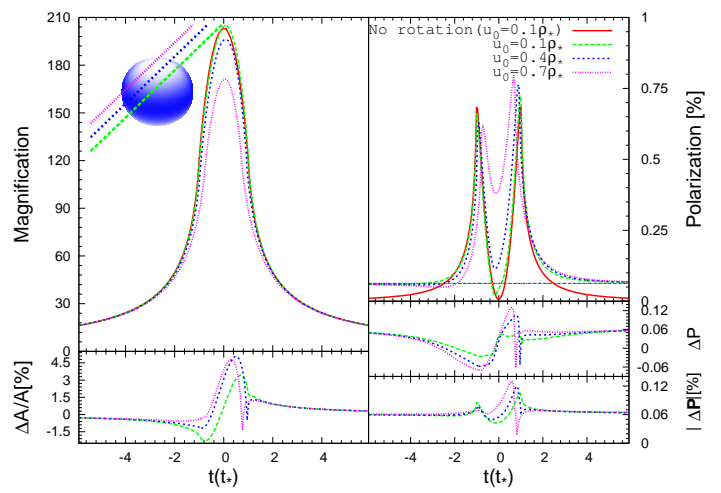

(a)

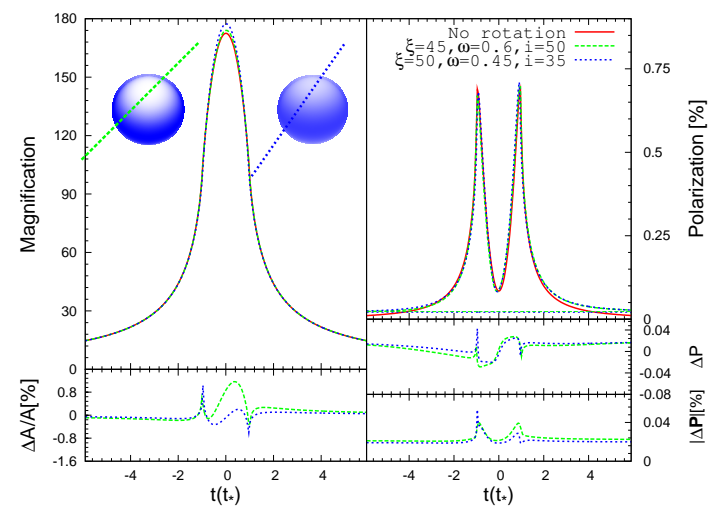

(c)

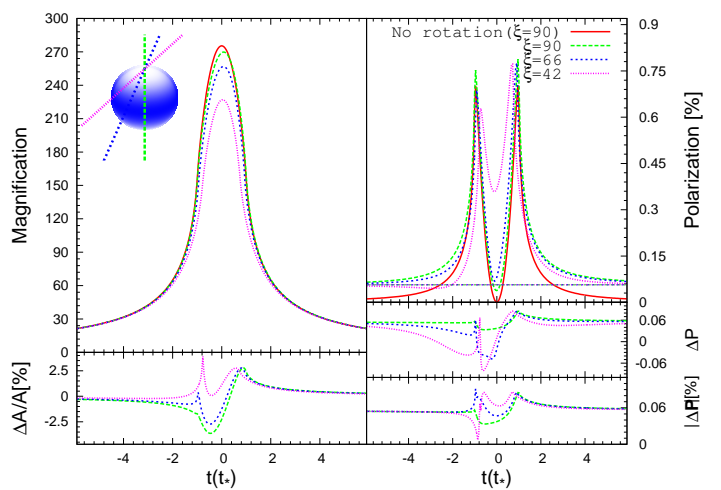

(b)

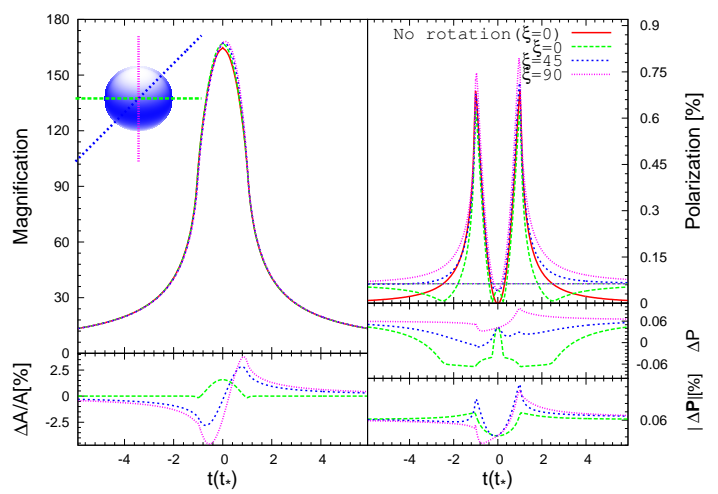

(d)

Figure 3. Example polarimetric microlensing events of elliptical source stars. In every subfigure, the light and polarimetric curves are shown in left and right panels. The source surface projected on the lens plane and the lens trajectory are shown with the inset in the left-hand panel. The map over the source surface represents stellar surface brightness. The simple models without the stellar rotation are plotted by red solid lines. The thinner straight lines in the right panel represent the intrinsic polarization signal of the elliptical source stars. The photometric and polarimetric residuals with respect to simple models are plotted in bottom panels. The parameters used to make these figures can be found in Table (1). We also set $\mathrm{M}_{1}=0.3 \mathrm{M}_{\odot}, \mathrm{D}_{1}=6.5 \mathrm{kpc}, \mathrm{D}_{\mathrm{s}}=8 \mathrm{kpc}$ and the limb-darkening coefficients over the source surface $\mathrm{c}_{1}=0.64$ and $\mathrm{c}_{2}=0.032$.

tainty in $\delta_{\mathrm{t}}$ is due to time intervals between consecutive polarimetry data points hypothetically taken during this time shift and their uncertainties.

In Figure 3(a) we plot the photometric and polarimetric light-curves of a microlensing event of an elliptical source for three different values of the lens impact parameter. In this Figure, the light and polarimetric curves are shown in the left and right panels respectively. The projected surface of the source star on the lens plane and lens trajectories are shown with an inset in the lefthand panel. The map over the source surface represents the surface brightness considering the gravity-darkening effect. The simple models without stellar rotation are shown by red solid lines. The thinner straight lines in right panel represent the intrinsic polarization signal of the elliptical source. The photometric and polarimetric residuals with respect to the simple models are plotted in bottom panels. The top polarimetric residual is the residual in the polarization degree $\Delta \mathrm{P}=\mathrm{P}^{\prime}-\mathrm{P}$ and the bottom one is the absolute value of the residual in the polar- ization vector $|\boldsymbol{\Delta} \boldsymbol{P}|=\sqrt{\mathrm{P}^{\prime 2}+\mathrm{P}^{2}-2 \mathrm{P}^{\prime} \mathrm{P} \cos 2\left(\theta_{\mathrm{p}}^{\prime}-\theta_{\mathrm{p}}\right)}$, where the prime symbol refers to the related quantity considering the stellar rotation. The parameters used to make this figure can be found in Table (1). We also set $\mathrm{M}_{\mathrm{l}}=0.3 \mathrm{M}_{\odot}, \mathrm{D}_{\mathrm{l}}=6.5 \mathrm{kpc}, \mathrm{D}_{\mathrm{s}}=8 \mathrm{kpc}, \mathrm{c}_{1}=0.64$ and $\mathrm{c}_{2}=0.032$. For each microlensing event, the radius of the spherical source of the simple model is equal to $\rho_{\star, \mathrm{s}}=\sqrt{\mathrm{u}_{0}^{2}+\mathrm{t}_{1}^{2}}$, i.e. the radius of the elliptical source where the lens is entering the source surface. Accordingly, the first peaks of polarimetry curves are coincided to the first peaks of simple models. The sharp peaks in polarimetry and photometry residuals while the lens is leaving the source surface represent the mentioned time shift and are owing to the ellipticity shape of the source surface which increases with enhancing the lens impact parameter.

If $\mathrm{u}_{0}>\rho_{\star}$ and for large inclination angles, the peak position of microlensing light curves of elliptical source stars does not significantly shift from the time of the 
Table 1

The parameters used to make microlensing events shown in Figures 3(a) 3(b) 3(c) 3(d) and (7).

\begin{tabular}{ccccccc}
\hline \hline & $\mathrm{M}_{\star}\left(\mathrm{M}_{\odot}\right)$ & $\mathrm{R}_{\mathrm{p}}\left(\mathrm{R}_{\odot}\right)$ & $\omega$ & $\mathrm{i}^{\circ}$ & $\mathrm{u}_{0}\left(\rho_{\star}\right)$ & $\xi^{\circ}$ \\
\hline $3(a)$ & 7.0 & 4.7 & 0.55 & 30.0 & -- & 43.9 \\
$3(b)$ & 5.0 & 3.6 & 0.5 & 25.0 & $0.9 \cos (\xi)$ & -- \\
$3(c)$ & 8.0 & 5.3 & -- & -- & 0.35 & -- \\
$3(d)$ & 9.0 & 5.8 & 0.55 & 30.0 & 0.0 & -- \\
$(7)$ & 3.8 & 2.9 & 0.50 & 15.0 & 0.43 & 42.0
\end{tabular}

Note. - The columns contains (i) the figure number, (ii) the mass of the source star $M_{\star}\left(M_{\odot}\right)$, (iii) the polar source radius $R_{p}\left(R_{\odot}\right)$, (iv) the angular speed of the source normalized to the break-up velocity $\omega,(\mathrm{v})$ the inclination angle of the stellar rotational axis with respect to the sky plane $\mathrm{i}^{\circ}$, (vi) the impact parameter of the lens trajectory with respect to the source center normalized to $\rho_{\star} \mathrm{u}_{0}\left(\rho_{\star}\right)$ and (vii) the angle of the lens trajectory with respect to the source semimajor axis $\xi^{\circ}$ respectively. We also set the lens mass $\mathrm{M}_{1}=0.3 \mathrm{M}_{\odot}$, the lens and source distances from the observer $D_{1}=6.5 \mathrm{kpc}$ and $\mathrm{D}_{\mathrm{s}}=8.0 \mathrm{kpc}$ for four first figures and $\mathrm{M}_{\mathrm{l}}=0.7 \mathrm{M}_{\odot}, \mathrm{D}_{\mathrm{l}}=4.1 \mathrm{kpc}$ and $\mathrm{D}_{\mathrm{s}}=8.2 \mathrm{kpc}$ for the last one. The limb-darkening coefficients are fixed at $c_{1}=0.64$ and $\mathrm{c}_{2}=0.032$

closest approach, since the maximum value of the Stokes intensity $\mathrm{I}_{\mathrm{I}}$ takes place in the stellar center (see equation 4 and the ellipticity affects on stellar limb points. On the contrary, in these bypass microlensing events the polarimetry peak positions vary from the time of the closest approach due to the ellipticity of the source surface, since the maximum value of the polarized Stokes intensity $I_{p}$ occurs at the stellar limb (see equation 4) and the stellar ellipticity also affects on these points. We expect that the peak position in polarimetric curves happens where the distance between the lens and the source edge minimizes. Accordingly, the time shift between polarimetric and photometric peaks is given by:

$$
\delta_{t}=\left(\alpha-\frac{1}{\alpha}\right) \frac{\cos \xi}{\sqrt{\alpha^{2}+\cot ^{2} \xi}} t_{\star} .
$$

The maximum value of this time shift happens when $\cot \xi=\sqrt{\alpha}$ which is equal to $0.33 \mathrm{t}_{\star}$ when $\alpha=1.5$. However, the gravity-darkening affects on this time shift when the inclination angle is small.

\subsection{Asymmetric perturbations owing to the gravity-darkening effect}

Gravity-darkening breaks the circular symmetry of the source surface brightness. However, gravity-darkening does not break the axial symmetry of the projected source surface with respect to its semiminor axis $\left(\mathrm{y}_{\mathrm{O}^{-}}\right.$ axis). This effect causes asymmetric perturbations in photometric and polarimetric light-curves of microlensing events, unless $\xi=0^{\circ}$ or $\mathrm{i}=90^{\circ}$. The gravitydarkening effect can be evaluated by the relative maximum deviation in the source surface brightness i.e. $\delta_{\mathrm{g}}$ which is given by:

$\delta_{g}=\left|\frac{g_{e f f}(\Omega, 0)-g_{e f f}\left(\Omega, 90^{\circ}\right)}{g_{\text {eff }}(\Omega, 0)}\right|=1-\frac{R_{p}^{2}}{R_{e q}^{2}}+\omega^{2}\left(\frac{2}{3}\right)^{3} \frac{\left.R_{\left(q_{2}\right.}\right)}{R_{p}}$

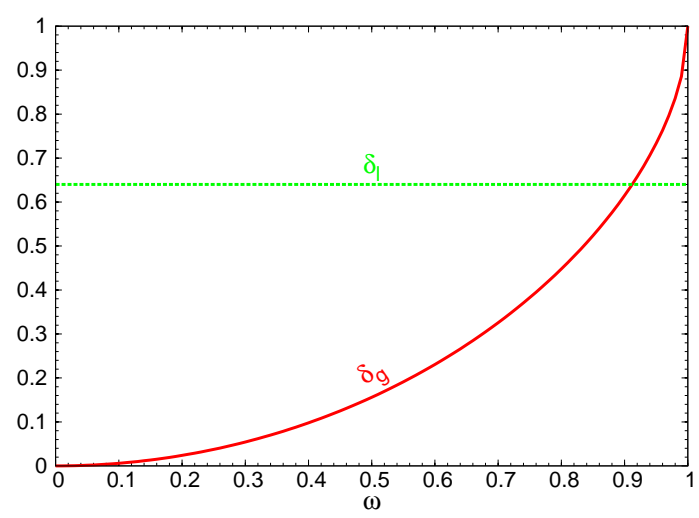

Figure 4. The maximum amounts of the relative deviations in the stellar surface brightness due to the gravity-darkening effect (red solid line) and owing to the limb-darkening effect (green dashed line) versus $\omega$.

which depends only on $\omega$ i.e. the stellar angular velocity normalized to the break-up velocity. On the other hand, the relative maximum deviation in the stellar surface brightness due to the limb-darkening effect is $\delta_{\mathrm{l}}=\mathrm{c}_{1}(=0.64)$. To compare the gravity-darkening and limb-darkening effects, we plot these relative maximum deviations in Figure (4), in which the red solid line represents $\delta_{\mathrm{g}}$ and the horizontal green dashed line shows $\delta_{l}$. Thus, the gravity-darkening effect generally is so smaller than the limb-darkening effect. It mostly makes small perturbations in microlensing curves, unless the lens crosses the projected position of the stellar rotation pole i.e. $\boldsymbol{d}_{\mathrm{R}}$. In these cases in which the lens impact parameters equal to $\mathrm{u}_{0}=\mathrm{R}_{\mathrm{p}} \cos (\mathrm{i}) \cos (\xi)$, the gravitydarkening effect can make detectable perturbations. The maximum deviation in microlensing light curves due to the gravity-darkening effect happens when $\xi=90^{\circ}$, since the maximum Stokes intensity $\mathrm{I}_{\mathrm{I}}$ which occurs at the source center (due to limb-darkening effect) is significantly perturbed by the gravity-darkening effect. The maximum polarimetric perturbation takes plane when $\tan (\xi)=\mathrm{R}_{\mathrm{p}} \cos (\mathrm{i}) / \mathrm{a}$, because in that case the lens trajectory crosses the stellar edge points which have the maximum value of $\delta_{\mathrm{g}}$ while these points own the largest polarized Stokes intensity. These asymmetric perturbations in polarimetry and photometry curves of microlensing events can be identified by comparing the left and right sides of these curves.

We show the maximum asymmetric perturbations in the light and polarimetry curves of a microlensing event due to the gravity-darkening effect in Figure 3(b). The characterizations of this plot are the same as those of Figure $3(\mathrm{a})$. We align the source trajectory so that it crosses the point $\boldsymbol{d}_{\mathrm{R}}$ for three different values of $\xi$. When $\xi=90^{\circ}$ the photometric residual due to the gravity-darkening effect maximizes. When $\xi=42^{\circ}$, the polarimetry residual becomes maximum. Asymmetry in microlensing curves due to the gravity-darkening effect is obvious, because the polarimetry and photometry residuals are not symmetric with respect to the time of the maximum magnification. Note that the sharp peaks when the lens is entering the source surface are due to the ellipticity ef- 


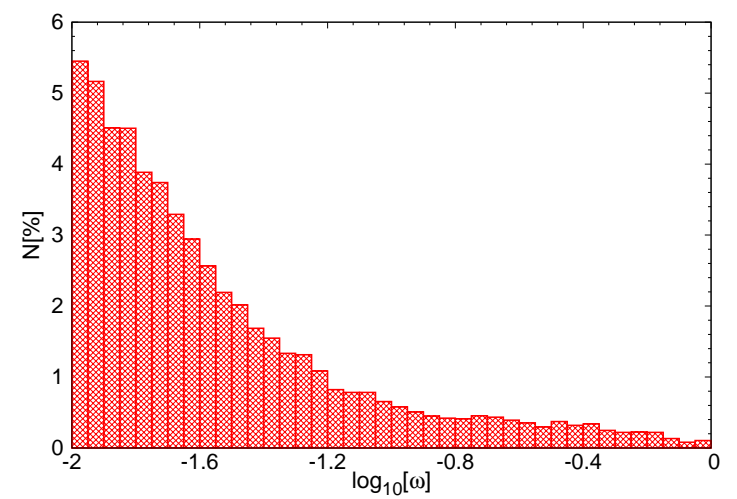

Figure 5. Histogram of $\omega$ in the logarithmic scale for the mainsequence stars observed by the Kepler satellite and their rotation periods were estimated by Rienhold et al. (2015).

fect and the mentioned time shift. However, the polarimetric deviations due to the gravity-darkening in this figure are less than the polarimetry precision of FORS2 which means higher quality polarimeters can realize these rotation-induced perturbations.

There is a problem in microlensing observations which is degeneracy. Even if microlensing observers correctly discern the type of the anomaly, all parameters of this anomaly can not be uniquely derived from the observed light or polarimetric curves. In polarimetry or photometry microlensing events of elliptical source stars this degeneracy exits. The intrinsic polarization signal of an elliptical source is a degenerate function of the inclination angle and the stellar angular velocity (see Figure 1). This degeneracy can not be resolved in microlensing observations. Figure 3(c) represents two different microlensing events of elliptical sources with different parameters, but the same polarimetry and photometry curves. These two microlensing events are degenerate. However, the microlensing degeneracy can even exit between different models with different kinds of anomalies. For example the microlensing curves and the intrinsic polarization signal of a rotating source star can be the same as those of two close binary source stars. This point is not studied in this work.

The polarization angle of an elliptical source (in our formalism) is zero with respect to its semimajor axis. The polarization angle of a lensed source star is $90^{\circ}$ with respect to the connection line of the source center and the lens position (see e.g. Sajadian \& Rahvar 2015). Hence, when the lens is entering the source surface with $\xi=0^{\circ}$ these polarization vectors are normal and in some time eliminate each other, so that the total polarization signal tends to zero at that time. Whereas, when $\xi=90^{\circ}$ these two polarization vectors are parallel and always magnify each other, so that the total polarization signal is ascending while the lens is entering the source surface. These points are shown in Figure 3(d) Detecting these features in the polarimetry curves of microlensing events helps to discern the angle between the lens trajectory and the semimajor axis of elliptical source which breaks the microlensing degeneracy. In this figure we set $\mathrm{u}_{0}=0$, so peak positions of polarimetry and photome-

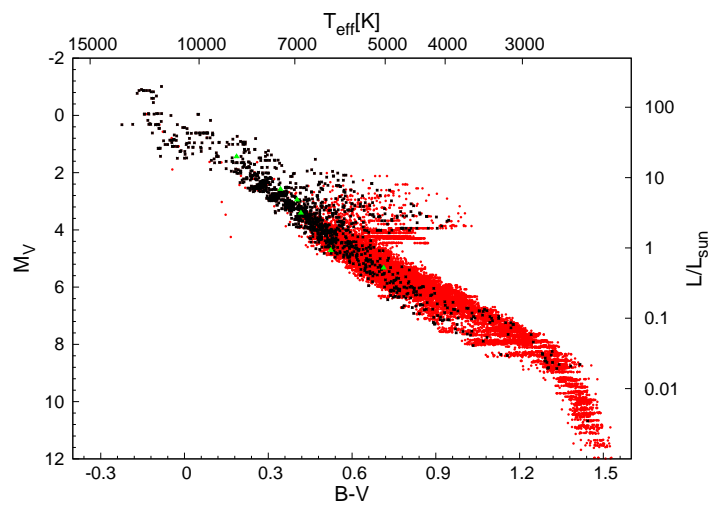

Figure 6. The Color-Magnitude diagram of the Kepler stars whose stellar rotational periods have been specified by Rienhold et al. (2013,2015) (red points). The stars with $\omega$ larger than 0.1 are indicated by black stars and the stars have the intrinsic polarization signals larger than $0.2 \%$ are represented by green triangles.

try curves have no time shift. However, there are some asymmetric perturbations due to the gravity-darkening effect. The photometric perturbation due to the ellipticity maximizes when $\xi=90^{\circ}$.

According to the different panels of Figure (3), it seems that most rotation-induced perturbations in polarimetric curves of microlensing events are less than the FORS2 accuracy i.e. $0.1 \%$. Hence, detecting the rotation-induced perturbations in the polarimetry microlensing curves can probably be done by the next-generation polarimeters with higher precisions than that of FORS2.

\section{OBSERVATIONAL REMARKS}

in the previous section we studied some aspects of polarimetry and photometry microlensing events of elliptical sources. In this section, we first investigate how per cent of the magnetically active stars observed by the Kepler satellite rotate fast and have the considerable values of $\omega$. The rotational periods of these stars were evaluated by their light curves by some groups (e.g. Reinhold et al. 2013, 2015 \& McQuillan et al. 2014). Then, we study whether the polarimetry observations by FORS2 of high-magnification microlensing events of fast rotating stars towards the Galactic spiral arms can give information on rotation-induced perturbations.

\subsection{Statistic of fast rotating stars based on the Kepler data}

The accurate statistics of fast rotating stars can not be fully determined owing to several limitations in observational methods. For example, the photometry method for measuring stellar rotational periods is sensitive to magnetically active stars with stellar spots. Also, the interferometry method evaluates the stellar oblateness of just nearby stars.

The Kepler satellite has provided stellar light curves of a very large sample of stars for more than four years by doing high-resolution uninterrupted photometry observations (Borucki et al. 2010; Koch et al. 2010). These data were analyzed to derive the stellar rotation periods and differential rotation effects by several authors, e.g. 


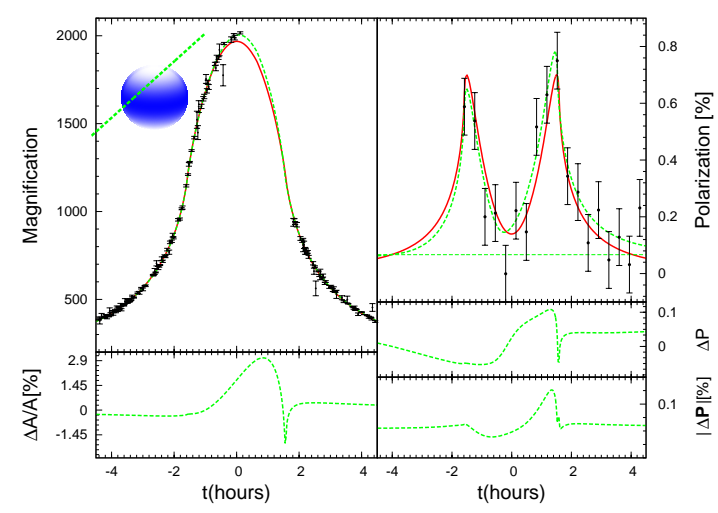

Figure 7. An example high-magnification polarimetry microlensing event and its simulated data points hypothetically taken by surveys and follow-up telescopes (over its light curve) and FORS2 polarimeter (over its polarimetry curve).

Reinhold et al. (2013,2015) analyzed a large sample of the Kepler stars to determine their rotation periods using different approaches based on the Lomb-Scargle periodogram. They first selected magnetically active stars which exhibit stellar spots at their surface, i.e. often main-sequence stars with $\log _{10}(\mathrm{~g})>3.5$. They noticed that $24.6 \%$ of stars in their sample were active. Using the stellar rotation periods given by Reinhold et al., the angular velocities of these active stars are inferred. We also estimate their critical velocities $\Omega_{\text {crit }}$ and as a result the ratio of the stellar angular velocity to the critical velocity $\omega$, according to the mass and radius of these stars given by Huber et al. (2014). The distribution of $\log _{10}(\omega)$ for this sample of stars is plotted in Figure (5). About 3.7 and 6.6 per cent of these stars have $\omega$ larger than 0.2 and 0.1 respectively. This sample just contains active main-sequence stars with $\log _{10}(\mathrm{~g})>3.5$, but not all unbiased stars.

The Color-Magnitude (CM) diagram of these Kepler stars is shown in Figure (6) (red points). In this figure, the stars with $\omega$ larger than 0.1 are shown with black stars. Most of fast rotating stars are early-type and hot stars. The green triangles represent the stars with the intrinsic polarization signals larger than $0.2 \%$, i.e. their polarization signals are measurable by FORS2 even without the lensing effect. For stars of this sample, we estimate their absolute magnitude using a synthetic CM diagram. We first generate a big ensemble of stars using the isochrones of Padova (Marigo et al. 2008) and according to the strategy explained in section (3) of Sajadian \& Rahvar (2012). We compare the temperature-luminosity diagram of stars in this sample with the related diagram of the generated synthetic sample of stars. For each star in our sample, we pick the characteristics of the most similar synthetic star in the generated ensemble.

In the next subsection, we simulate high-magnification microlensing of the stars specified by the black stars in Figure (6) to study if rotation-induced perturbations are discernable through hypothetical polarimetry and photometry observations of these events.

\subsection{Monte Carlo simulation}

It is well known that massive and hot stars rotate very fast, whereas most main-sequence or red giant stars have small or moderate rotational speeds (e.g. Bouvier 2013). The Galactic bulge often contains old and cold stars while the Galactic disk stars have a wide range of ages (e.g. Ortolani et al. 1995, Russeil 2003). Indeed, our galaxy seems to have two spiral arms containing old stars and four spiral arms including gas and young stars (Urquhart et al. 2014). Although, the microlensing optical depth toward the Galactic spiral arms is less than that toward the Galactic bulge by one order of magnitude (Rahal et al. 2009), the mean duration of microlensing events in these directions is $\sim 60$ days (Rahal et al. 2009) which is longer than that toward the Galactic bulge, i.e. $\sim 27$ days (Wyrzykowski et al. 2014). Thus, the time scale of crossing the source radius by the lens, i.e. $t_{\star}$ toward the spiral arms is about twice that toward the Galactic bulge. We note that $t_{\star}$ is the polarimetry time scale during a microlensing event. On the whole, the Galactic spiral arms are more suitable to be probed for finding stellar rotation effects. Hence, we simulate high-magnification microlensing events of those source stars whose rotational properties studied by Reinhold et al. toward the Galactic spiral arms. We choose the Carina-Sagittarius arm and in the direction $1=300^{\circ}$ and $\mathrm{b}=-1^{\circ}$. Indeed, we assume that the stellar local population probed by the Kepler satellite is representative of the galactic population. This hypothesis is most probably justified for stars in the Galactic disk.

We have two criteria for selecting these stars as source stars: (a) the stars with $\omega>0.1$ and (b) those brighter than 21 mag in I-band after being located at that Galactic arm. These criteria decrease the number of possible source stars to 773 . We finally investigate the possibility of discerning polarimetry and photometry rotationinduced perturbations in these high-magnification microlensing events.

The generic procedure for a Monte Carlo simulation as well as the used distribution functions to determine the mass of lenses, the velocities of both sources and lenses and the distribution of matter in the Galaxy to determine the lens and source positions toward the Galactic arm were described in our previous works (Sajadian 2014, Sajadian 2015b: Moniez et al. 2016) and we do not repeat them here. Also, we use the Galactic extinction model in three dimensions developed by Marshall et al. (2006). We consider only high-magnification events with the lens impact parameter less than the threshold value of 0.001 . The inclination angle of the rotational axis for each source star is uniformly chosen in the range of $\left[0,90^{\circ}\right]$.

We assume these events are observed by FORS2 which reaches the highest polarimetry precision $\sigma_{\mathrm{p}}=0.1 \%$. The necessary $\mathrm{S} / \mathrm{N}$ to achieve this precision is $1 / \sigma_{\mathrm{p}}=1000$ for the imaging polarimetry mode (IPOL). We generate synthetic data points hypothetically taken by FORS2 over every polarimetry curve. In this regard, we calculate the necessary exposure time to achieve the highest polarimetric accuracy. The definition of $\mathrm{S} / \mathrm{N}$ can be found in Sajadian (2015b). In addition to the exposure time, there are two extra waste times due to the retarder waveplate rotation and CCD readout. This overhead time lasts about $18 \mathrm{~min}$ for each polarimetry data 

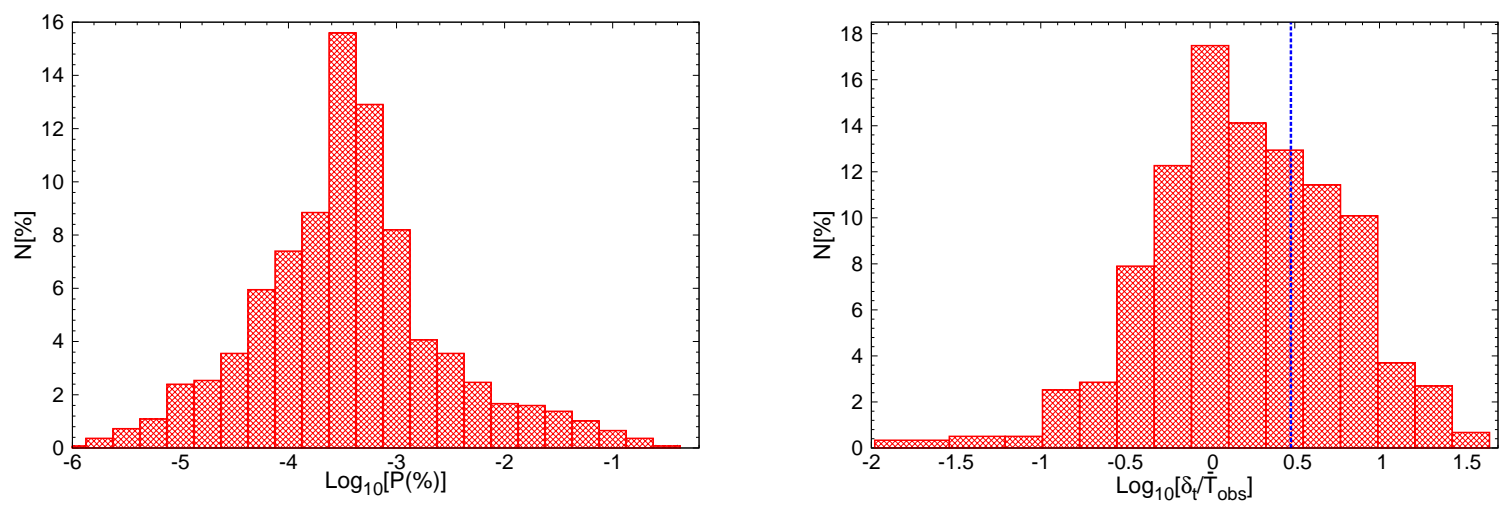

Figure 8. The results of the Monte Carlo simulation: Left panel: the histogram of the intrinsic polarization signals of source stars. Right panel: the histogram of the time shifts induced by the stellar ellipticity in the polarimetry peak positions $\delta_{\mathrm{t}}$ normalized to the averaged observational time for taking each polarimetry data point $\overline{\mathrm{T}}_{\text {obs }}$. This quantity shows the possible number of polarimetric data points during the rotation-induced time shifts. The blue dashed line shows the amount $\delta_{\mathrm{t}} / \overline{\mathrm{T}}_{\mathrm{obs}}=3$. Both of them are plotted in the logarithmic scale.

point by FORS2. In this regard, all details are explained in subsection (3.1). The start time of observation by FORS2 is chosen randomly in the range of $[-3.0: 3.0] \mathrm{t}_{\star}$ and after about 6 hours, the observation is interrupted until the next night (after 18 hours). For all simulated events, we set $t_{0}=0$.

For generating photometry data points, we use the sampling and the photometric uncertainties (i.e. $\sigma_{\mathrm{a}}$ ) taken by some archived high-magnification events around the peak of their light curves. We use the highmagnification microlensing events given by Choi et al. (2012). The simulated (photometry and polarimetry) data points are shifted with respect to the model light and polarimetric curves according to their photometric and polarimetric uncertainties by Gaussian functions. One of simulated light and polarimetry curves of highmagnification microlensing events and its synthetic data points are shown in Figure (7). Its parameters can be found in Table (1). Also we put $\mathrm{t}_{\mathrm{E}}=70$ days and $\rho_{\star}=0.001$. Then, we investigate the simulated photometry and polarimetry curves to verify if the rotationinduced perturbations of source stars are distinguishable. There are three tests in this regard:

(a) If the intrinsic polarization signal of a rotating source star is larger than $0.2 \%$, twice the polarimetric accuracy of the FORS2 polarimeter, the polarization signal can be measured even without the lensing effect. The distribution of the intrinsic polarization signals of source stars is shown in the left panel of Figure (8). Six source stars have the net polarization signals more than $0.2 \%$. These stars are indicated in Figure (6) by green triangles.

(b) As discussed in the previous section, the stellar rotation breaks the symmetry of polarimetry and photometry microlensing curves with respect to $t_{0}$. This anomaly shifts (i) the position of the second polarimetry peak with respect to the symmetric position of the first polarimetry peak in transit microlensing events and (ii) the time position of the polarimetry peak with respect to the photometry peak position in bypass cases. For simulated microlensing events, we calculate these time shifts i.e. $\delta_{\mathrm{t}}$. The ratio of this time shift to the mean value of the time interval between two consecutive polarimetric data points gives the possible number of polarimet- ric data points that can be taken during this time shift. The histogram of this quantity in the logarithmic scale, $\log _{10}\left[\delta_{t} / \overline{\mathrm{T}}_{\text {obs }}\right]$, is plotted in the right panel of Figure (8). The number of events which have $\delta_{\mathrm{t}} / \overline{\mathrm{T}}_{\text {obs }}>3$ is 221 , i.e. about $28.6 \%$ of the total number of simulated events. Detecting this time shift needs enough polarimetric data points at the polarimetry peak position(s). We assume that real positions of photometric peaks can be inferred by fitting process. The number of simulated events for which FORS2 has observed the polarimetry peak(s) is 188 , about $24.3 \%$ of events. On the whole, $4.7 \%$ of the simulated events have $\delta_{\mathrm{t}} / \bar{\tau}_{\mathrm{p}}>3$ as well as their polarimetry peaks are covered by synthetic data points, which means that during the time shift $\delta_{\mathrm{t}}$ more than three polarimetry data points are taken by FORS2. However, for discerning these perturbations, the polarimetry residual during this time shift with respect to the not rotating (spherical) star model should be more than at least $2 \sigma_{\mathrm{p}}$, which is probable according to fast fall off polarimetry microlensing curves around their peaks.

(c) Finally, we can discern stellar rotation effects on polarimetry and photometry microlensing curves by detecting asymmetric perturbations which are mostly due to gravity-darkening. In that case, for each simulated event we calculate the $\Delta \chi^{2}=\chi_{\mathrm{e}}^{2}-\chi_{\mathrm{s}}^{2}$ in which $\chi_{\mathrm{e}}^{2}$ and $\chi_{\mathrm{s}}^{2}$ are resulted from fitting the real model with the elliptical source star and the simple model with the not rotating (spherical) source star to the simulated data points respectively. The parameters of the simple model are similar to the parameters used to make the real model, except the source star does not rotate. Also, the radius of the spherical source star in simple models equals to the radius of the source star where the lens is entering the source surface, i.e. $\rho_{\star, \mathrm{s}}=\sqrt{\mathrm{u}_{0}^{2}+\mathrm{t}_{1}^{2}}$ in transit microlensing events and $\rho_{\star, \mathrm{s}}=\sqrt{\mathrm{ab}} \mathrm{x}_{\mathrm{rel}} / \mathrm{R}_{\mathrm{E}}$ in bypass events. The distributions of $\Delta \chi^{2}$ in the logarithmic scale from fitting to photometry data points (red dashed histogram) and to polarimetry data points (blue histogram) are plotted in Figure (9). In about 83.1 and 0.1 per cent of simulated light and polarimetry microlensing curves (respectively), the values of $\Delta \chi^{2}$ are higher than 150 . The small number of events for which $\Delta \chi_{\mathrm{p}}^{2}>150$ means that (i) FORS2 


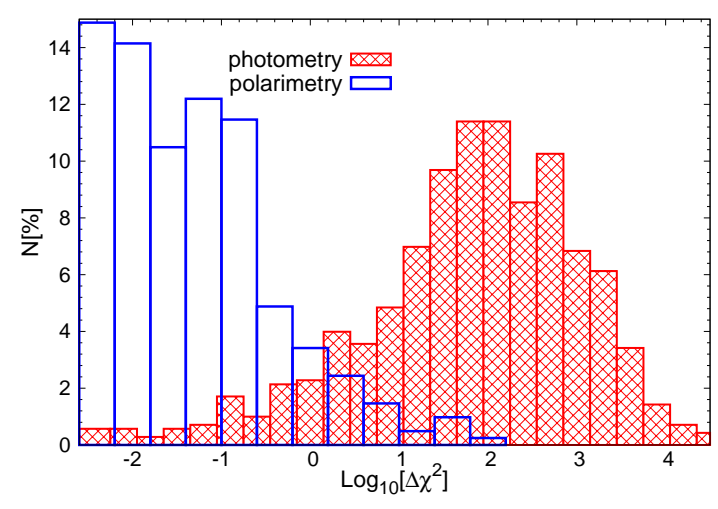

Figure 9. The distributions of $\Delta \chi^{2}=\chi_{\mathrm{e}}^{2}-\chi_{\mathrm{s}}^{2}$ in the logarithmic scale, where $\chi^{2} \mathrm{~s}$ with the indies e and $\mathrm{s}$ are resulted from fitting the real models of elliptical source stars and the simple models of spherical source stars respectively, to photometry (red dashed histogram) and polarimetry data points (blue histogram). For each simulated event, the parameters used to generate the simple model are similar to the parameters used to make the real model without considering the stellar rotation.

by itself can not more likely cover polarimetry curves of high-magnification microlensing events as well as (ii) its polarimetry precision is too low to distinguish rotationinduced perturbations.

Considering all of the mentioned tests for discerning rotation-induced perturbations, we conclude that in 37 and 642 simulated events (which contribute 4.8 and 83.1 per cent of all simulated events) the polarimetry and photometry perturbations induced by stellar rotations are distinguishable respectively. Although, the photometry observation is more efficient than the polarimetry one in detecting the stellar rotation effects, but by doing only photometry observation we can not realize the time shift in the photometry peak position, whereas this time shift is so helpful in discerning the anomaly kind. However, the small polarimetry efficiency for detecting stellar rotation effects is rather owing to the lack of enough number of polarimetry data points to cover the polarimetry peak(s), if we assume that these observations are done by FORS2 by itself.

\section{SUMMARY AND CONCLUSIONS}

The stellar rotation causes the ellipticity and gravitydarkening effects which break the spherical symmetry of the source surface and the circular symmetry of its surface brightness respectively. Accordingly, a rotating star has a net polarization signal whose intensity depends on the inclination angle of the rotational axis as well as the stellar angular velocity. For fast rotating stars $(\omega>0.6)$ the magnitude of this polarization signal is comparable with the polarization signal in the transit microlensing events. This anomaly should be considered in the polarimetry microlensing calculations of these stars allowing to characterize the elliptical properties of the source stars. The intrinsic polarization signals for slow or moderate rotating stars with $\omega<0.6$ that are too small to be measured by themselves, may be highlighted during a high-magnification microlensing event.

Polarimetry and photometry curves in single-lens mi- crolensing events of spherical (non rotating) source stars have symmetric shapes with respect to the time $t_{0}$ of the closest approach . The stellar ellipticity breaks this symmetry and causes time shifts $\delta_{\mathrm{t}}$ in (i) the position of the second polarimetry peak with respect to the symmetric (with respect $t_{0}$ ) position of the first peak in transit microlensing events and (ii) the position of the polarimetry peak with respect to the photometry peak position in bypass cases. However, when $\xi=0^{\circ}, \xi=90^{\circ}$ or $\mathrm{u}_{0}=0$ there is no time shift, because of the symmetric shape of elliptical source surface projected on the sky plane with respect to its semimajor and minor axes.

Gravity-darkening produces asymmetric perturbations in polarimetry and photometry curves of microlensing events. These perturbations maximize whenever the lens trajectory crosses the projected position of the stellar rotation pole on the sky plane. In that case, if its angle with respect to the source semimajor axis equals to $\xi=90^{\circ}$ the photometric perturbation maximizes and if $\xi=\arctan \left(\mathrm{R}_{\mathrm{p}} \cos (\mathrm{i}) / \mathrm{R}_{\mathrm{eq}}\right)$ the polarimetric perturbation becomes maximum.

The intrinsic polarization signal of an elliptical source is a degenerate function of the inclination angle and the stellar angular velocity (see Figure 1). Hence, different elliptical source stars with the same intrinsic polarization signals can have the same polarimetry and photometry microlensing curves.

In order to study and compare the photometry and polarimetry efficiencies for detecting the stellar rotation effects in high-magnification microlensing events toward the Galactic spiral arms, we simulated them and considered the fast rotating stars observed by the Kepler satellite $(\omega>0.1)$ as the source stars. We generated syntectic (polarimetry and photometry) data points hypothetically taken by FORS2 and survey and follow-up telescopes (respectively) for each microlensing event. About $0.5 \%$ of the source stars had the intrinsic polarization signals greater than $0.2 \%$ which can potentially be measured by FORS2 directly. In $4.7 \%$ of total simulated events more than three polarimetry data points were taken during the time shift $\delta_{\mathrm{t}}$ by FORS2. In these events, the rotation-induced time shift and as a result the ellipticity of the source surface projected on the sky plane can likely be measured. We also investigated whether asymmetric perturbations in simulated polarimetry and photometry curves due to the gravity-darkening effect can be inferred, by calculating the difference between the values of $\chi_{\mathrm{e}, \mathrm{s}}^{2}$ from fitting (a) the real microlensing models of elliptical source stars and (b) simple microlensing models of not rotating (spherical) source stars to synthetic data points. Almost 83.1 and 0.1 per cent of photometry and polarimetry curves had $\Delta \chi^{2}$ greater than 150 respectively.

On the whole, polarimetry and photometry perturbations due to the stellar rotation were detectable in 4.8 and 83.1 per cent of all simulated events respectively. Therefore, the stellar rotation signatures in high-magnification microlensing events of early-type stars are mostly realizable through photometry observations with the technology presently available. Although, the photometry observation is more efficient than the polarimetry one for detecting stellar-induced anomalies, but by only doing photometry observations we can not discern the time 
shift in photometry peak positions resulted from the stellar ellipticity. This time shift is so helpful to realize the nature of the anomaly and obtain some information about the ellipticity of the projected source surface. However, the small polarimetry efficiency for detecting stellar rotation effects is owing to: (i) the low polarimetry precision of FORS2 and (ii) the long necessary observational time for taking one polarimetry data point by FORS2 (in comparison with the polarimetry time scales of microlensing events) which results insufficient number of data points to cover the polarimetry peak(s). This time shift can likely be distinguished by high-quality polarimeters of the next generation.

I acknowledge T. Reinhold and S. Bognulo for useful discussions and comments and also J.-Y., Choi for providing the data of some high-magnification microlensing events. I thank the referee for helpful comments and suggestions which significantly improved the manuscript. Finally, I am grateful to Sh. Baghram and H.Ghodsi for good comments to improve text of paper. This work was supported by a grant (94017434) from the Iran National Science Foundation (INSF).

\section{REFERENCES}

Abney W. D., 1877, MNRAS, 37, L278.

Affer L., Micela G., Favata F. \& Flaccomio E., 2012, MNRAS, 424, L11.

Al-Malki M. B., Simmons J. F. L., Ignace R., Brown J. C., \& Clarke D., 1999, A \& A, 347, L919.

Barnes S. A., 2007, ApJ, 669, L1167.

Bjorkman J. E. \& Bjorkman K. S., 1994, ApJ, 436, L818.

Bouvier J., 2013, EAS Publications Series, 62, L143.

Borucki, W. J., Koch, D., Basri, G., et al. 2010, Sci, 327, L977.

Carciofi A. C., \& Bjorkman J. E., 2006, ApJ, 639, L1081.

Carciofi A. C., et al., 2006, ApJ, 652, L1617.

Casas R., Vaquero J. M., \& Vazquez M., 2006, Sol. Phys., 234, L379.

Chandrasekhar S., 1960, Radiative Transfer. Dover Publications, New York.

Choi J.-Y., Shin I.-G., Park S.-Y., et al. 2012, ApJ, 751, 41

Churchwell E.D., Babler B. L., Meade M. R. et al., 2009, PASP, $121,213$.

Collins G.W. II, Harrington J. P. 1966, ApJ, 146, L152.

Durney B. R., \& Latour J., 1978, Geophysical and Astrophysical Fluid Dynamics, 9, L241.

Ejeta C., Boehnhardt H., BAgnulo S. \& Tozzi G. P. 2012, A \& A, 537, A23.

Fluri D.M., Stenflo J. O., 1999, A \& A, 341, L902.

Gallet F. \& Bouvier J., 2013, A \& A, 556, L36.

Gaudi B. S. \& Haiman Z., 2004, (arXiv:astro-ph/0401035v1).

Gould A., 1997, ApJ, 483, L98.

Harrington J.P. \& Collins G. W., 1968, ApJ, 151, L1051.

Heyrovský D. \& Loeb A., 1997, ApJ, 490, L38.
Huber, D., Silva Aguirre, V., Matthews, J. M., et al., 2014, ApJS, 211, L2.

Ignace R., Al-Malki M.B., Simmons J. F.L., Brown J. C., Clarke D. \& Carson J. C., 2009, A \& A, 496, L503.

Ingrosso G., Calchi Novati S., De Paolis F., et al. 2012, MNRAS, 426, L1496.

Ingrosso G., Calchi Novati S., De Paolis F., et al. 2015, MNRAS, 446, L1090.

Irwin J., Berta Z.K., Burke C.J., et al., 2011, ApJ, 727, 56.

Kervella P., Thévenin F., Di Folco E. \& Ségransan D., 2004, a \& A, 426, L297.

Kitchatinov L. L., 2005, Actra Astron, 57, 149.

Koch, D. G., Borucki, W. J., Basri, G., et al., 2010, ApJL, 713, L79.

Kraft, R. P., 1970, Spectroscopic Astrophysics. An Assessment of the Contributions of Otto Struve, L385.

Kroupa, P., Tout, C. A., \& Gilmore, G. 1993, MNRAS, 262, L545.

Kroupa, P. 2001, MNRAS, 322, L231.

Le Bouquin J.-B., Absil O., Benisty M., et al., 2009, A \& A, 498, L41.

Maeder A. \& Meynet G., 2011, arXiv:1109.6171

Maoz D. \& Gould A., 1994, ApJ, 425, L67.

Moniez M., Rahvar S., Sajadian S., Karami M. \& Ansari R. 2016, in preperation.

Marigo P., Girardi L., Bressan A., Groenewegen M. A. T., Silva L., Granato G. L., 2008, A \& A, 482, L883.

McAlister H. A., ten Brummelaar T. A., et al., 2005, ApJ, 628, L439.

McQuillan A., Aigrain S., \& Mazeh T., 2013, MNRAS, 432, L1203.

Lebovitz N.R., 1967, ARA \& A, 5, L465.

Ortolani S., Renzini A., Gilmozzi R. et al. 1995, Nature, 377, L701.

Peterson D. M., et al., 2004, SPIE, 5491, L65.

Rahal, Y. R., Afonso C., Albert J.-N., et al. 2009, A \& A, 500, L1027.

Reinhold T., Reiners A. \& Basri G., 2013, A \& A, 560, L4.

Reinhold T. \& Gizon L., 2015, A \& A, .....

Robin, A. C., Reylé, C., Derrière, S., Picaud, S., 2003, A \& A, 409, L523.

Russeil D., 2003, A \& A, 397, 133.

Sajadian S., 2014, MNRAS, 439, L3007.

Sajadian S., 2015a, AJ, 149, L147.

Sajadian S., 2015b, MNRAS, 452, L2587.

Schatzman, E., 1962, Annales d'Astrophysique, 25, L18.

Scholz A. \& Eislöffel J., 2004, A \& A, 419, L249.

Scholz A. \& Eislöffel J., 2005, A \& A, 429, L1007.

Schneider P., Wagoner R. V., 1987, ApJ, 314, L154.

Simmons J.F.L., Bjorkman J.E., Ignace R., Coleman I.J., 2002, MNRAS, 336, 501

Skumanich, A., 1972, ApJ, 171, L565.

Stamford P. A. \& Watson R. D., 1980, Acta Astron, 30, L193.

Tinbergen J., 1996, Astronomical Polarimetry. Cambridge Univ. Press, New York.

von Zeipel, H., 1924, MNRAS, 84, L665.

Urquhart, J. S., Figura, C. C., Moore, T. J. T., Hoare, M. G., et al. 2014, MNRAS, 437, 1791.

Whitney B.A., et al., 2013, ApJS, 207, L30.

Wyrzykowski, Ł., et al., 2014, arXiv:1405.3134 